\title{
Lista de Avaliadores Ad hoc - Ano 2013
}

\begin{tabular}{|c|c|}
\hline Avaliador Ad hoc & Instituição de Vínculo \\
\hline Adriano José Pereira & Universidade Federal de Santa Maria \\
\hline André Moreira Cunha & Universidade Federal do Rio Grande do Sul \\
\hline Angélica Massuquetti & Universidade do Vale do Rio dos Sinos \\
\hline Augusta Pelinski Raiher & Universidade Estadual de Ponta Grossa \\
\hline Carlos Aguiar de Medeiros & Universidade Federal do Rio de Janeiro \\
\hline César Mattos & Consultoria Legislativa da Câmara dos Deputados \\
\hline Clailton Ataides de Freitas & Universidade Federal de Santa Maria \\
\hline Cristiano Aguiar de Oliveira & Universidade Federal do Rio Grande \\
\hline Daniela Magalhães Prates & Universidade Estadual de Campinas \\
\hline Eduardo Pontual Ribeiro & Universidade Federal do Rio de Janeiro \\
\hline Erik Figueiredo & Universidade Federal do Rio Grande do Norte \\
\hline Eugênio Lagemann & Universidade Federal do Rio Grande do Sul \\
\hline Fernando de Holanda Barbosa & Fundação Getúlio Vargas - Rio de Janeiro \\
\hline Fernando Ferrari Filho & Universidade Federal do Rio Grande do Sul \\
\hline Flavio Fligenspan & Universidade Federal do Rio Grande do Sul \\
\hline Flávio Vilela Vieira & Universidade Federal de Uberlândia \\
\hline Flávio Ziegelmann & Universidade Federal do Rio Grande do Sul \\
\hline Giuliano Contento Oliveira & Universidade Estadual de Campinas \\
\hline Hermógenes Saviani Filho & Universidade Federal do Rio Grande do Sul \\
\hline Janice Castro & Universidade Federal do Rio Grande do Sul \\
\hline Jean Max Tavares & Pontifícia Universidade Católica de Minas Gerais \\
\hline Jefferson Andronio Staduto & Universidade Estadual do Oeste do Paraná \\
\hline Jorge Eduardo Soromenho & Universidade de São Paulo \\
\hline Leonardo Monastério & Universidade Católica de Brasília \\
\hline Leonardo Xavier da Silva & Universidade Federal do Rio Grande do Sul \\
\hline Luciano Moraes Braga & Instituto Brasileiro de Geografia e Estatística \\
\hline Luiz Paulo Nogueról & Universidade de Brasília \\
\hline Marcelo Antonio Conterato & Universidade Federal do Rio Grande do Sul \\
\hline Marcelo Milan & Universidade Federal do Rio Grande do Sul \\
\hline Marcelo Curado & Universidade Federal do Paraná \\
\hline Marcilene Martins & Universidade Federal do Rio Grande do Sul \\
\hline Marcos Eugênio da Silva & Universidade de São Paulo \\
\hline Maria Mollo & Universidade de Brasília \\
\hline
\end{tabular}




\begin{tabular}{|l|l|}
\hline \multicolumn{1}{|c|}{ Avaliador Ad hoc } & \multicolumn{1}{c|}{ Instituição de Vínculo } \\
\hline Marta dos Reis Castilho & Universidade Federal do Rio de Janeiro \\
\hline Mauricio Vaz Lobo Bittencourt & Universidade Federal do Paraná \\
\hline Nelson Seixas dos Santos & Universidade Federal do Rio Grande do Sul \\
\hline Octavio Conceição & Universidade Federal do Rio Grande do Sul \\
\hline Orlando Martinelli & Universidade Federal de Santa Maria \\
\hline Paulo Dabdab Waquil & Universidade Federal do Rio Grande do Sul \\
\hline Pedro Fonseca & Universidade Federal do Rio Grande do Sul \\
\hline Raul Assumpção Bastos & Fundação de Economia e Estatística \\
\hline Regina Madalozzo & Instituto de Ensino e Pesquisa - Insper \\
\hline Rosana Ribeiro & Universidade Federal de Uberlândia \\
\hline Ronald Hillbrecht & Universidade Federal do Rio Grande do Sul \\
\hline Sabino Porto Jr. & Universidade Federal do Rio Grande do Sul \\
\hline Sérgio Monteiro & Universidade Federal do Rio Grande do Sul \\
\hline Simone Silva de Deos & Universidade Estadual de Campinas \\
\hline
\end{tabular}

